\title{
ENVIRONMENTALLY INDUCED CHANGES IN RIBOSOMAL RNA CISTRON NUMBER: PURPORTED LACK OF CORRELATION WITH PHENOTYPE
}

\author{
R. FRANKHAM* \\ Institute of Animal Genetics, West Mains Road, Edinburgh EH9 3JN
}

Received 21.i.77

IT is of crucial importance to genetics and to its practical applications to establish whether the cause(s) of environmental induction of heritable change is of Lamarckian origin or whether it is explicable in terms of presently known genetic mechanisms.

In his interesting paper on the environmental induction of heritable changes in flax Cullis (1976) asserts in his summary that "There was no correlation between ribosomal cistron number and either nuclear DNA content of the genotrophs or plant weight". If true, this statement would suggest that changes at the rRNA locus were not any part of the causation of induction of heritable change in flax. However, in the case of plant weight Cullis' assertion is clearly not supported by the data he presents in his table 1 and statistical analyses of his data confirms this, the rank correlations between ribosomal cistron number and plant weight being $0 \cdot 78$. The data are not normally distributed due to selection of the material on plant weight (Cullis, pers. comm.) so it is most appropriate to measure the significance of association between ribosomal cistron number and plant weight using the regression coefficient (lack of correlation implies zero covariance and results in both a zero correlation and a zero regression). Plant weights are only given as large, small or intermediate, but if these are coded as 2, 0 , and 1 , respectively, the regression coefficient of ribosomal cistron number on plant weight is $524 \cdot 7 \pm 113.5$ and is highly significantly different from zero $(\mathrm{P}<0 \cdot 01)$. Consequently the correlation between ribosomal RNA cistron number and plant weight is not zero. It should be noted that Timmis and Ingle (1975) obtained very similar results to Cullis (1976) but in contrast concluded that " the changes described in rRNA gene redundancy may be a primary event in the induction of heritable changes in flax". An alternative statistical treatment of Cullis' data, using the analysis of variance, yields a highly significant effect of plant weight class on ribosomal cistron number $\left(\mathrm{F}_{2,6}=31 \cdot 02\right)$ and a highly significant effect of Large versus Small plant weight classes $\left(F_{1,6}=46.95\right)$, so the comments above do not depend critically on the form of statistical analysis used.

If some of the plant material was chimaeric, then the author could also be led to spurious conclusions as he has assayed only rDNA levels in leaves and total nuclear DNA in apical buds. The reason for this suggestion is that transitory chimaeric states, when material is in the process of change, are to be expected with a number of models for induction. In fact, the large variation in ribosomal cistron number among extracts from $\mathrm{L}_{\mathbf{3}}$, found

* Permanent address: School of Biological Sciences, Macquarie University, North Ryde, N.S.W. 2113, Australia. 
by Timmis and Ingle (1975) could be due to some of the material being chimaeric. They commented that "the reason for the greater variation in $\mathrm{L}_{3}$ may be that some plants are about to change to a small phenotype". Consequently, the work of Timmis and Ingle (1975), Cullis (1976) and other work on correlations between various features of environmentally induced heritable changes in flax, must be interpreted with caution in the absence of evidencc that chimaeras are not involved.

Acknowledgments.-I wish to thank Dr C. A. Cullis for his comments on an carlier draft of this Note and Dr W. G. Hill for comments and advice.

\section{References}

CULLIS, c. A. 1976. Fnvironmentally induced changes in ribosomal RNA cistron number in flax. Heredity, 36, 73-79.

TrMmis, J. N., AND INGLE, J. 1975. The status of ribosomal RNA genes during nuclear DNA reversion in flax. Biochem. Genet., 13, 629-634. 